\title{
Absolute Valued Algebras with Strongly One Sided Unit
}

\author{
Alassane Diouf ${ }^{1}$ \\ ${ }^{1}$ Département de Mathématiques et Informatiques, Faculté des Sciences et Techniques, Université Cheikh Anta Diop, \\ Dakar, Sénégal
}

Correspondence: Alassane Diouf, Département de Mathématiques et Informatiques, Faculté des Sciences et Techniques, Université Cheikh Anta Diop, Dakar, Sénégal. E-mail: dioufalassane@ @otmail.fr

Received: October 30, 2016 Accepted: December 5, 2016 Online Published: December 30, 2016

doi:10.5539/jmr.v9n1p32

URL: http://dx.doi.org/10.5539/jmr.v9n1p32

\begin{abstract}
We classify the absolute valued algebras with strongly left unit of dimension $\leq 4$. Also we prove that every 8-dimensional absolute valued algebra with strongly left unit contain a 4-dimensional subalgebra, next we determine the form of theirs algebras by the duplication process.
\end{abstract}

Keywords: absolute valued algebra, strongly left unit, duplication process

Mathematics Subject Classification: 17A35, 17A36

\section{Introduction}

The absolute valued algebras are introduced by Ostrowski 1918. It's the normed algebra $A$ such that $\|x y\|=\|x\|\|y\|$ for all $x, y$ in $A$. For an element $a$ in an algebra A, we denote by $L_{a}: A \rightarrow A x \mapsto a x$ and $R_{a}: A \rightarrow A x \mapsto x a$. The algebra is called division if and only if $R_{a}$ and $L_{a}$ are bijective for all $a$ in $A$. We denote by $O$ the orthogonal group of linear isometries of Euclidean space $\mathbb{H}$. We recall $O^{+}$the subgroup of proper linear isometries and $O^{-}$the subset of improper linear isometries. Let $A$ be an absolute valued algebra with unit, then $A$ is isomorphic to $\mathbb{R}, \mathbb{C}, \mathbb{H}$ or $\mathbb{O}$ (Urbanik \& Wright, 1960). The absolute valued algebras with left unit satisfying to $\left(x^{2}, x^{2}, x^{2}\right)=0$, for all $x \in A$ is classified in (Diankha \& all, 20132). These algebras are finite dimensional and isomorphic to $\mathbb{R}, \mathbb{C},{ }^{\star} \mathbb{C}, \mathbb{H},{ }^{\star} \mathbb{H},{ }^{\star} \mathbb{H}(i, 1), \mathbb{O},{ }^{\star} \mathbb{O},{ }^{\star} \mathbb{O}(i, 1), \widetilde{\mathbb{O}}$ or $\widetilde{\mathbb{O}}(i)$ and the element $e$ satisfy to $L_{e}=R_{e}^{2}=I_{A}$. The algebras $\mathbb{H}_{i}, \mathbb{O}_{i}$ (Diankha \& all, 2013 $)$, satisfy to $L_{e}=R_{e}^{2}=I_{A}$ and not satisfy to $\left(x^{2}, x^{2}, x^{2}\right)=0$. In this paper we give a classification of the absolute valued algebras with strongly left unit of dimension $\leq 4$. We proves that if $A$ is 8-dimensional absolute valued algebra with strongly left unit, then $A$ contain a 4-dimensional subalgebra and $A$ is obtained by the duplication process. Otherwise $A$ is of the form $\mathbb{H} \times \mathbb{H}_{(\varphi, \psi)}$ with $\varphi, \psi: \mathbb{H} \rightarrow \mathbb{H}$ are linear isometries such that $\varphi(1)=1$ and $(\varphi, \psi)^{2}=(\varphi, \psi)$. The algebras $\mathbb{R}, \mathbb{C},{ }^{\star} \mathbb{C}, \mathbb{H},{ }^{\star} \mathbb{H}, \mathbb{H}_{i},{ }^{\star} \mathbb{H}(i, 1)$, $\mathbb{O},{ }^{\star} \mathbb{O}, \mathbb{O}_{i},{ }^{\star} \mathbb{O}(i, 1), \widetilde{\mathbb{O}}$ are absolute valued algebras with strongly left unit. This list is completed by new algebras.

\section{Preliminary}

In this section we recall the some interest results:

Theorem 1 The finite-dimensional absolute valued real algebras with a left unit are precisely those of the form $\mathbb{A}_{\varphi}$, where $\mathbb{A} \in\{\mathbb{R}, \mathbb{C}, \mathbb{H}, \mathbb{O}\}$ and $\varphi$ is an isometric of the euclidien espace $\mathbb{A}$ fixes 1 , and $\mathbb{A}_{\varphi}$ denotes the absolute-valued real algebra obtained by endowing the normed space of $\mathbb{A}$ with the product $x \odot y:=\varphi(x) y$. Moreover, given linear isometries $\varphi, \phi: \mathbb{A} \rightarrow \mathbb{A}$ fixing 1 , the algebras $\mathbb{A}_{\varphi}$ and $\mathbb{A}_{\phi}$ are isomorphic if and only if there exists an algebra automorphism $\psi$ of $\mathbb{A}$ satisfying $\phi=\psi \circ \varphi \circ \psi^{-1}$ (Rochdi, 2003).

Lemma 1 Let $A$ be an absolute valued algebra with strongly left unit. The following equalities hold for all $x \in A$.

1. $[(x e) x] e=x(x e)$

2. $[x(x e)] e=(x e) x$

3. $[x e, x]=<e, x>[e, x-x e]$

If, moreover, $x$ is orthogonal to $e$, then

4. $[x e, x]=0$

5. $(x e) x^{2}=2<e, x^{2}>x-\|x\|^{2} x e$

6. $(x e)^{2}=2<e, x^{2}>e-x^{2}$

7. $x^{2} x=-\|x\|^{2} x e$ (Chandid \& Rochdi, 2008). 
The group $G_{2}$ acts transitively on the sphere $S(\operatorname{Im}(\mathbb{O})):=S^{6}$, that is the mapping $G_{2} \rightarrow S^{6} \Phi \mapsto \Phi(i)$ is surjective (Postnikov, 1985).

Let $\mathbb{A}$ be one of the unital absolute valued algebras $\mathbb{R}, \mathbb{C}, \mathbb{H}$ of dimension $m$. Consider the caley dickson product $\odot$ in $\mathbb{A} \times \mathbb{A}$, we define on the space $\mathbb{A} \times \mathbb{A}$ the product

$$
(x, y) \star\left(x^{\prime}, y^{\prime}\right)=\left(f_{1}(x), f(x)\right) \odot\left(g_{1}\left(x^{\prime}\right), g\left(y^{\prime}\right)\right) .
$$

With $f_{1}, g_{1}, f, g$ be linear isometries of $\mathbb{A}$ and $f_{1}(1)=g_{1}(1)=1$. We obtain a $2 m$-dimensional absolute valued real algebra $\mathbb{A} \times \mathbb{A}_{\left(f_{1}, f\right),\left(g_{1}, g\right)}$. The process is called duplication process. Note that the algebra is left unit if $g_{1}=g=I_{\mathbb{A}}$ and this case we not the algebra by $\mathbb{A} \times \mathbb{A}_{\left(f_{1}, f\right)}$. We have the following result (Calderon \& all, 2011):

Theorem 2 Let A be an 8-dimensional absolute valued algebra, then the following are equivalent:

1. A contains a 4-dimensional subalgebra.

2. A is obtained by the duplication process.

3. Aut(A) contains a reflexion.

Lemma 2 Let $\mathcal{I}^{+}=\left\{f \in O^{+}: f\right.$ involutive $\}, \mathcal{I}^{-}=\left\{f \in O^{-}: f\right.$ involutive $\}, \mathcal{I}_{1}^{+}=\left\{f \in \mathcal{I}^{+}: f(1)=1\right\}$ and $\mathcal{I}_{1}^{-}=\left\{f \in \mathcal{I}^{-}\right.$: $f(1)=1\}$. We have:

1. $O^{+}=\left\{T_{a, b}: a, b \in S(\mathbb{H})\right\}$

2. $O^{-}=\left\{T_{a, b} \circ \sigma_{\mathbb{H}}: a, b \in S(\mathbb{H})\right\}:=O^{+} \circ \sigma_{\mathbb{H}}$

3. $O_{1}^{+}=\left\{T_{a, \bar{a}}: a \in S(\mathbb{H})\right\}$

4. $O_{1}^{-}=\left\{T_{a, \bar{a}} \circ \sigma_{\mathbb{H}}: a \in S(\mathbb{H})\right\}:=O_{1}^{+} \circ \sigma_{\mathbb{H}}$

5. $\mathcal{I}^{+}=\left\{ \pm I_{\mathbb{H}}\right\} \cup\left\{T_{a, b}: a, b \in S(\operatorname{Im}(\mathbb{H}))\right\}$

6. $\mathcal{I}^{-}=\left\{ \pm T_{a, \bar{a}}: a \in S(\mathbb{H})\right\}$

7. $\mathcal{I}_{1}^{+}=\left\{I_{\mathbb{H}}\right\} \cup\left\{T_{a, \bar{a}}: a \in S(\operatorname{Im}(\mathbb{H}))\right\}$

8. $\mathcal{I}_{1}^{-}=\left\{\sigma_{\mathbb{H}}\right\} \cup\left\{T_{a, \bar{a}} \circ \sigma_{\mathbb{H}}: a \in S(\operatorname{Im}(\mathbb{H}))\right\}:=\mathcal{I}_{1}^{+} \circ \sigma_{\mathbb{H}}($ Diankha $\mathcal{E}$ all, 2013 $)$.

Corollary 1 Let $A$ be an absolute valued algebra with left unit satisfying to $\left(x^{p}, x^{q}, x^{r}\right)=0$ with $\{p, q, r\} \in\{1,2\}$. Then $A$ contains a strongly left unit.

Proof. Lemma 1 (Diankha \& all, 2013 2 ) and proof of Proposition 4.8 (Chandid \& Rochdi, 2008)..

The converse of Corollary 1 is false, an effect the algebra $A:=\mathbb{O}_{i}$ is an absolute valued algebra with strongly left unit and $A$ not satisfy to $\left(x^{2}, x^{2}, x^{2}\right)=0$.

\section{Absolute Valued Algebras with Strongly Left Unit}

Definition 1 An element $e \in A$ is called strongly left unit, if it's left unit and square root of right unit $\left(L_{e}=R_{e}^{2}=I_{A}\right)$.

Theorem 3 Let $A$ be an absolute valued algebra with strongly left unit. Then $A$ is finite dimensional. Moreover if $\operatorname{dim}(A) \leq 4$, then $A$ is isomorphic to $\mathbb{R}, \mathbb{C},{ }^{\star} \mathbb{C}, \mathbb{H},{ }^{\star} \mathbb{H}, \mathbb{H}(i, 1)$ or ${ }^{\star} \mathbb{H}(i, 1)$.

Proof. The algebra $A$ is left unit, hence $A$ is left division (Rodriguez, 2004). Morover the assertion $R_{e}^{2}=I_{A}$ imply that $A$ is right division, then $A$ is finite dimensional. Also $A$ is of the form $\mathbb{A}_{\varphi}$, with $\varphi$ a linear isometric fixed 1 and $\mathbb{A} \in\{\mathbb{R}, \mathbb{C}, \mathbb{H}, \mathbb{O}\}$ (Theorem 1). If $\operatorname{dim}(A) \leq 2$, it's clear that $A$ is isomorphic to $\mathbb{R}, \mathbb{C}$ or ${ }^{\star} \mathbb{C}$.

Assume now $\operatorname{dim}(A) \geq 4$, then the assertion $R_{e}^{2}=I_{A}$ imply:

$$
\begin{aligned}
x & =(x \odot 1) \odot 1 \\
& =\varphi^{2}(x) .
\end{aligned}
$$

Then $\varphi$ is an involutive linear isometric $\varphi^{2}=I_{\mathbb{A}}$.

If $\operatorname{dim}(A)=4$, we have:

$\varphi \in \mathcal{I}_{1}^{+} \cup \mathcal{I}_{1}^{-}=\left\{I_{\mathbb{H}}\right\} \cup\left\{T_{a, \bar{a}}: a \in S(\operatorname{Im}(\mathbb{H}))\right\} \cup\left\{\sigma_{\mathbb{H}}\right\} \cup\left\{T_{a, \bar{a}} \circ \sigma_{\mathbb{H}}: a \in S(\operatorname{Im}(\mathbb{H}))\right\}$ (Lemma 2). 
- If $\varphi=I_{\mathbb{H}}$, then $A$ is isomorphic to $\mathbb{H}$.

- If $\varphi=\sigma_{\mathbb{H}}$, then $A$ is isomorphic to ${ }^{\star} \mathbb{H}$.

- If $\varphi=T_{a, \bar{a}}: a \in S(\operatorname{Im}(\mathbb{H}))$, there exist $v \in S(\mathbb{H})$ such that $v a \bar{v}=i$ and let the automorphism $\Phi=T_{v, \bar{v}}$ of $\mathbb{H}$ with $\Phi^{-1}=T_{\bar{v}, v}$, we have $\Phi \circ T_{a, \bar{a}} \circ \Phi^{-1}=T_{i, \bar{i}}$. Then $A$ is isomorphic to $\mathbb{H}_{T_{i, \bar{i}}}$ (Theorem 1) and the map $\Phi: \mathbb{H}(i, 1) \rightarrow \mathbb{H}_{T_{i, \bar{i}}}$ $x \mapsto x i$ is an isomorphism algebras.

- If $\varphi=T_{a, \bar{a}} \circ \sigma_{\mathbb{H}}: a \in S(\operatorname{Im}(\mathbb{H}))$, there exist $u \in S(\mathbb{H})$ such that $u a \bar{u}=i$ and let the automorphism $\Phi=T_{u, \bar{u}}$ of $\mathbb{H}$, we have:

$$
\begin{aligned}
\Phi \circ T_{a, \bar{a}} \circ \sigma_{\mathbb{H}} \circ \Phi^{-1} & =T_{u, \bar{u}} \circ T_{a, \bar{a}} \circ \sigma_{\mathbb{H}} \circ T_{\bar{u}, u} \\
& =T_{u, \bar{u}} \circ T_{a, \bar{a}} \circ T_{\bar{u}, u} \circ \sigma_{\mathbb{H}} \\
& =T_{u a \bar{u}, u \overline{a u}} \circ \sigma_{\mathbb{H}} \\
& =T_{i, \bar{i}} \circ \sigma_{\mathbb{H}} .
\end{aligned}
$$

Then $A$ is isomorphic to $\mathbb{H}_{T_{i, \bar{i}} \circ \sigma_{\mathbb{H}}}$ (Theorem 1) and the map $\Phi:^{\star} \mathbb{H}(i, 1) \rightarrow \mathbb{H}_{T_{i, \bar{i}} \circ \sigma_{\mathbb{H}}} x \mapsto \bar{i} x$ is an isomorphism of algebras.. If $\operatorname{dim}(A)=4$, the last result can be obtained so by using the identity $R_{e}^{2}=I_{\mathbb{H}}$ and the principal isotopes of $\mathbb{H}$ : $\mathbb{H}(a, 1)$, ${ }^{\star} \mathbb{H}(a, 1)$, where $a \in S(\mathbb{H})$. For the first isotope $e=\bar{a}$, and for the second isotope $e=a$.

For all alternative algebra $A$, Artin's theorem (Schafer, 1996) shows that for any $x, y \in A$, the set $\{x, y, \bar{x}, \bar{y}\}$ is contained in an associative subalgebra of $A$. We note by $T(x)=x+\bar{x}$ the tace of $x \in A$ and we have $x^{2}-T(x) x+\|x\|^{2} e=0$ for all $x \in A$. As $A$ is real alternative quadratic algebra, we have $A=\mathbb{R} e \oplus \operatorname{Im}(A)$ (Frobenius decomposition) and their exist a unique linear form $\lambda: A \rightarrow \mathbb{R}$ such that $\lambda(1)=1, \operatorname{ker}(\lambda)=\operatorname{Im}(A)$ and $\langle x, y\rangle=\lambda(x \bar{y})=\lambda(\bar{x} y)$ for all $x, y \in A$ (*) (Koecher \& Remmert, 1991). Otherwise for all $x, y \in \operatorname{Im}(A)$ we have $x y+y x=-2<x, y\rangle e(\star)$ and the identity $x y x=2 \lambda(x y) x-\|x\|^{2} \bar{y}$ for all $x, y \in \operatorname{Im}(A)$ is called the triple product identity (TPI).

In 8-dimensional, by the duplication process we recover theirs algebras.

Theorem 4 Let A be an 8-dimensional absolute valued algebra with strongly left unit. Then A contains a four-dimensional subalgeba.

Proof. We have $\mathbb{O}=\mathbb{R} \oplus \operatorname{Im}(\mathbb{O})$ and their exist a unique linear form $\lambda: \mathbb{O} \rightarrow \mathbb{R}$ such that $\lambda(1)=1$ and $\operatorname{ker}(\lambda)=\operatorname{Im}(\mathbb{O})$. Let $u \in 1^{\perp}$, we have $0=<1, u>=<\Phi^{n}(1), \Phi^{n}(u)>=<1, \Phi^{n}(u)>$. Then we have $\varphi^{n}\left(1^{\perp}\right) \subseteq 1^{\perp}$, for all $n \in \mathbb{N}$. The algebra $A$ is of the form $\mathbb{O}_{\Phi}$ with $\Phi(1)=1$ and $\Phi^{2}=I_{\mathbb{O}}$ (Theorem 1 and Theorem 3). Otherwise we have $i \odot i=\Phi(i) i$ and $i \odot 1=\Phi(i)$. Using the equality $(\star)$ we have $i \Phi(i)+\Phi(i) i=-2<i, \Phi(i)>1$. Also using Lemma 1 (7), we have $\Phi[\Phi(i) i]=\Phi(i) i$. Using the TPI we have

$$
\Phi(i) i \Phi(i)=2 \lambda[\Phi(i) i] \Phi(i)+i=-2<i, \Phi(i)>\Phi(i)+i .
$$

and

$$
i \Phi(i) i=2 \lambda[i \Phi(i)] i+\Phi(i)=-2<i, \Phi(i)>i+\Phi(i) .
$$

Hence we have the products,

$$
\begin{aligned}
& \Phi(i) \odot 1=\Phi^{2}(i)=i . \\
& \Phi(i) \odot i=\Phi^{2}(i) i=-1 \\
& \Phi(i) \odot \Phi(i)=\Phi^{2}(i) \Phi(i)=i \Phi(i)=-2<i, \Phi(i)>1-\Phi(i) i . \\
& \Phi(i) \odot \Phi(i) i=\Phi^{2}(i) \Phi(i) i=i \Phi(i) i=-2<i, \Phi(i)>i+\Phi(i) . \\
& \Phi(i) i \odot 1=\Phi[\Phi(i) i]=\Phi(i) i \\
& \Phi(i) i \odot i=\Phi[\Phi(i) i] i=\Phi(i) i^{2}=-\Phi(i) . \\
& \Phi(i) i \odot \Phi(i)=\Phi[\Phi(i) i] \Phi(i)=\Phi(i) i \Phi(i)=-2<i, \Phi(i)>\Phi(i)+i \\
& \Phi(i) i \odot \Phi(i) i=\Phi[\Phi(i) i] \Phi(i) i=(\Phi(i) i)^{2}=T[\Phi(i) i] \Phi(i) i-1 .
\end{aligned}
$$




\begin{tabular}{ccccc}
\hline$\odot$ & 1 & $i$ & $\Phi(i)$ & $\Phi(i) i$ \\
\hline 1 & 1 & $i$ & $\Phi(i)$ & $\Phi(i) i$ \\
\hline$i$ & $\Phi(i)$ & $\Phi(i) i$ & -1 & $-i$ \\
\hline$\Phi(i)$ & $i$ & -1 & $-2<i, \Phi(i)>1-\Phi(i) i$ & $-2<i, \Phi(i)>i+\Phi(i)$ \\
\hline$\Phi(i) i$ & $\Phi(i) i$ & $-\Phi(i)$ & $-2<i, \Phi(i)>\Phi(i)+i$ & $T[\Phi(i) i] \Phi(i) i-1$ \\
\hline
\end{tabular}

Then the algebra $A$ contains a four-dimensional sub-algebra..

Theorem 5 Let $A$ be an 8-dimensional absolute valued algebra with strongly left unit. Then $A$ is of the form $\mathbb{H} \times \mathbb{H}_{(\varphi, \psi)}$ where $(\varphi, \psi)$ are linear isometries of $\mathbb{H}$ belong to $\mathbb{S}_{1} \cup \mathbb{S}_{2} \cup \mathbb{S}_{3} \cup \mathbb{S}_{4}$ with:

$$
\begin{aligned}
& \mathbb{S}_{1}=\left\{I_{\mathbb{H}}\right\} \times\left\{ \pm I_{\mathbb{H}}, T_{a, b}: a, b \in S^{2}, \pm T_{c, \bar{c}} \circ \sigma_{\mathbb{H}}: c \in S^{3}\right\} \\
& \mathbb{S}_{2}=\left\{\sigma_{\mathbb{H}}\right\} \times\left\{ \pm I_{\mathbb{H}}, T_{a, b}: a, b \in S^{2}, \pm T_{c, c} \circ \sigma_{\mathbb{H}}: c \in S^{3}\right\} \\
& \mathbb{S}_{3}=\left\{T_{a, \bar{a}}: a \in S^{2}\right\} \times\left\{ \pm I_{\mathbb{H}}, T_{b, c}: b, c \in S^{2}, \pm T_{d, \bar{d}} \circ \sigma_{\mathbb{H}}: d \in S^{3}\right\} \\
& \mathbb{S}_{4}=\left\{T_{a, \bar{a}} \circ \sigma_{\mathbb{H}}: a \in S^{2}\right\} \times\left\{ \pm I_{\mathbb{H}}, T_{b, c}: b, c \in S^{2}, \pm T_{d, \bar{d}} \circ \sigma_{\mathbb{H}}: d \in S^{3}\right\} .
\end{aligned}
$$

Proof. Using the Theorem 2 and Theorem 4, the algebra $A$ is obtained by the duplication process. It's clear that the algebra $A$ is of the form $\mathbb{H} \times \mathbb{H}_{(\varphi, \psi)}$, with $\varphi(1)=1$. The linear isometric $(\varphi, \psi)$ is involitive, then $\varphi^{2}=\psi^{2}=I_{A}$. We have $\varphi \in \mathcal{I}_{1}^{+} \cup \mathcal{I}_{1}^{-}$and $\psi \in \mathcal{I}^{+} \cup \mathcal{I}^{-}$. Then the lemma 2 gives the result..

Problem 1 In dimension 8, it will be interesting to specify these algebras by reducing the isomorphism classes.

\section{Acknowledgements}

I thank the reviewers for the relevant remarks and suggestions.

\section{References}

Albert, A. A. (1947). Absolute valued real algebras. Ann. Math., 48, 495-501.

Calderon, A., Kaidi, A., Martin, C., Morales, A., Ramirez, M., \& Rochdi, A. (2011). Finite - dimensional absolute valued algebras. israel journal of mathematics, 184, 193 - 220.

Chandid, A. \& Rochdi, A. (2008). A survey on absolute valued algebras satisfying $\left(x^{i}, x^{j}, x^{k}\right)=0$. Int. J. Algebra, 2, 837-852.

Diankha, O., Diouf, A., \& Rochdi, A. (2013 $)$. A brief statement on the absolute-valued algebras with one-sided Unit. Int. J. Algebra, 7(17), 833-838.

Diankha, O., Diouf, A., Ramirez, M. I., \& Rochdi, A. $\left(2013_{2}\right)$. Absolute-valued algebras with one-sided unit satisfying $\left(x^{2}, x^{2}, x^{2}\right)=0$. Int. J. Algebra, 7(19), 935-958.

Koecher, M., \& Remmert. (1991). Numbers. Springer Verlag.

Postnikov, M. (1985), Leçons de Géométrie. Groupes et algèbres de Lie. Editions Mir.

Rochdi, A. (2003). Eight-dimensional real absolute valued algebras with left unit whose automorphism group is trivial. IJMMS, 70, 4447-4454.

Rodriguez, A. (1992). One-sided division absolute valued algebras. Publ. Math., 36,925-954.

Rodriguez, A. (2004). Absolute valued algebras, and absolute valuable Banach spaces. Advanced courses of mathematical analysis I, 99-155, World Sci. Publ., Hackensack, NJ.

Ramirez, M. I. (1999). On four absolute valued algebras. Proceedings of the International Conference on Jordan Structures (Malaga, 1997), 169-173.

Schafer, R. (1996). An Introduction to Nonassociative Algebras. Academic Press.

Urbanik, K., \& WrightfF. B. (1960). Absolute valued algebras. Proc. Amer. Math. Soc. 11, 861-866.

\section{Copyrights}

Copyright for this article is retained by the author(s), with first publication rights granted to the journal.

This is an open-access article distributed under the terms and conditions of the Creative Commons Attribution license (http://creativecommons.org/licenses/by/4.0/). 